\title{
Caracterización de las investigaciones financiadas con fondos concursables en la UNAH, 2013-2017
}

Lesbia Jeannette Buitrago ${ }^{1}$

\section{RESUMEN}

Este artículo analiza las investigaciones realizadas en la UNAH con fondos concursables entre los años 2013 y 2017. Se analiza la metodología más empleada en las investigaciones elaboradas por Facultades y Centros Regionales. Así como los temas prioritarios los que más se ha investigado y finaliza con la descripción de la realización de las investigaciones por género, año, Facultad y/o Centro Regional, de un total de 239 investigaciones realizadas en el período indicado. Es un estudio cuantitativo con alcance descriptivo.

Entre los hallazgos destacados están que las investigaciones financiadas se han enmarcado mayoritariamente en los temas prioritarios de la UNAH números 8 (Cultura, Ciencia y Educación por un lado); y 9 (Salud, Estado y Sociedad). Las investigaciones financiadas por la UNAH durante dicho período se caracterizan por ser mayoritariamente de enfoque cuantitativo y realizadas por mujeres; a las que se suman las realizadas por equipos en los que participaron ambos géneros.

Palabras clave: investigación, metodología, género, temas prioritarios

\footnotetext{
1 Jefa del Departamento Proyectos de Investigación, Dirección de Investigación Científica UNAH: investigacionunah.proyectos.lb@gmail.com
} 


\section{ABSTRACT}

This article analyzes the research carried out at the UNAH with competitive funds between 2013 and 2017. The methodology most used in the research carried out by faculties and regional centers is analyzed. As well as the priority topics, the most researched and finalized with the description of the realization of the research by gender, year, faculty and / or regional center, out of a total of 239 investigations carried out in the indicated period. It is a quantitative study with descriptive scope.

Among the outstanding findings are that the funded research has been framed mainly in the priority issues of the UNAH numbers 8 (Culture, Science and Education on the one hand); and 9 (Health, State and Society). The research financed by the UNAH during this period is characterized by being mainly of a quantitative approach and carried out by women; to which are added those made by teams in which both genders participated.

Keywords: research, methodology, gender, priority themes. 


\section{INTRODUCCIÓN}

La UNAH apoya la investigación junto a la docencia y la vinculación como una de las funciones básicas como institución rectora de la educación superior protagonista en la transformación de la sociedad nacional. La universidad ha tomado acciones para institucionalizar la investigación, y una de ellas es la asignación de fondos concursables dirigidas a sus profesores y estudiantes; los primeros como parte de su quehacer académico y para enriquecer la docencia y retroalimentarla con la vinculación así como la resolución de problemas de la sociedad, los sectores productivos y sociales. En el caso de los estudiantes como parte de su formación profesional.

Los fondos para la investigación son asignados mediante un concurso de participación para estudiantes de grado y posgrado, así como de profesores que laboran en la institución. Estos fondos están disponibles para las diez facultades y los ocho centros regionales, se concursan por medio de dos convocatorias al año. Estas convocatorias de investigación siguen un proceso de evaluación metodológica por cada uno de los proyectos presentados; dicho proceso culmina con las notificaciones oficiales a quienes aprobaron el concurso, dicha aprobación se logra con un puntaje obtenido arriba de un $90 \%$ en la evaluación total.

El propósito de este artículo es identificar los enfoques metodológicos utilizados por los investigadores por año. según Facultad; Centro Regional, género, así como también los temas que más han sido estudiados de acuerdo a los Temas prioritarios establecidos en la UNAH entre 2013 y 2017.

\section{METODOLOGÍA}

La metodología empleada para este análisis es cuantitativa, el alcance es descriptivo y la población de estudio han sido 239 investigaciones realizadas en la UNAH con fondos concursables entre los años que comprenden desde el 2013 hasta el 2017.

\section{Becas de investigación por temas prioritarios}

Las investigaciones financiadas no tienen restricciones en cuanto al uso del enfoque metodológico a emplear ni las técnicas a utilizar para la recolección de datos, pero deben estar en concordancia con los trece temas prioritarios establecidos por la 
UNAH (2015-2019), los que están a su vez articulados con los ejes del Plan de Nación, el Consejo Superior Universitario Centroamericano (CSUCA) y los Objetivos de Desarrollo Sostenible (ODS). Se describen los enfoques utilizados por los investigadores por cada Facultad y Centro Regional, las técnicas de mayor uso entre los años 2013 y 2017.

Los ejes y temas prioritarios de investigación de la UNAH (2016-2019) se dividen en cuatro ejes los cuales a su vez completan trece temas:

a) Desarrollo económico y social, que engloba los temas de infraestructura y desarroIlo territorial; pobreza e inequidad; y globalización, productividad y competitividad.

b) Democracia y gobernabilidad, que incluye reforma del Estado; democracia, ciudadanía y participación; y conflictividad política y social.

c) Población y condiciones de vida, que comprende población, necesidades básicas y transición demográfica; cultura, ciencia y educación; salud, Estado y sociedad; y seguridad y violencia.

d) Ambiente, biodiversidad y desarrollo contiene cambio climático y vulnerabilidad; seguridad alimentaria y nutricional; y desarrollo energético: fuentes, impacto y política.

\section{RESULTADOS DEL ANÁLISIS}

\section{a) Temas investigados:}

Como puede observarse en el gráfico 1, los temas No. 8 y 9 son los mayoritarios en el abordaje de las investigaciones en el período 2013-2017.

El tema prioritario No.3 ha tenido un aumento en las investigaciones pues pasó de 8.1\% en 2013 a 14.3\% en 2017; Este tema incluye: globalización: impacto y desafíos; productividad y competitividad; mercado interno y externo; crisis económica y financiera; innovación y tecnología; cultura productiva y gestión empresarial; turismo sostenible y gestión y conservación del patrimonio (cultural y natural) y cadenas productivas y de valor. En cambio, el tema prioritario No. 2 registró el $13.5 \%$ de las investigaciones en 2013, presentando una disminución en los años 2014 con 2.0\%, en 2015 con $2.1 \%$, en 2016 con $1.8 \%$ y en 2017 no se aplicó a este tipo de investigación. Este tema prioritario incluye aspectos como: determinantes de la pobreza y la inequidad; nuevas propuestas metodológicas de medición de la pobreza y la inequidad; políticas públicas de combate a la pobreza y la inequidad (evaluación de impac- 
to); riqueza cultural y natural versus pobreza e inequidad territorial; servicios básicos, pobreza e inequidad; pobreza, inequidad y costo social de la ineficacia y corrupción pública y grupos vulnerables, pobreza e inequidad.

El tema prioritario No. 8 Cultura, ciencia y educación tuvo un significativo incremento de $2013(8.1 \%)$ a 2014 (51.0\%), aunque disminuyó en 2015 (23.4\%) volviendo a aumentar en 2016 (31.6\%) y 2017 (34.7\%). Incluye aspectos como: eficacia, eficiencia y pertinencia del sistema educativo; tecnología e innovación educativa; Investigación y enseñanza en ciencia y tecnología; sistema educativo nacional; procesos de reforma educativa; interculturalidad y multiculturalidad; territorio, identidad y cultura; diversidad étnica y lingüística. (DICyP, 2015)

Gráfico 1. Becas de investigación por temas prioritarios de la UNAH 2013-2017

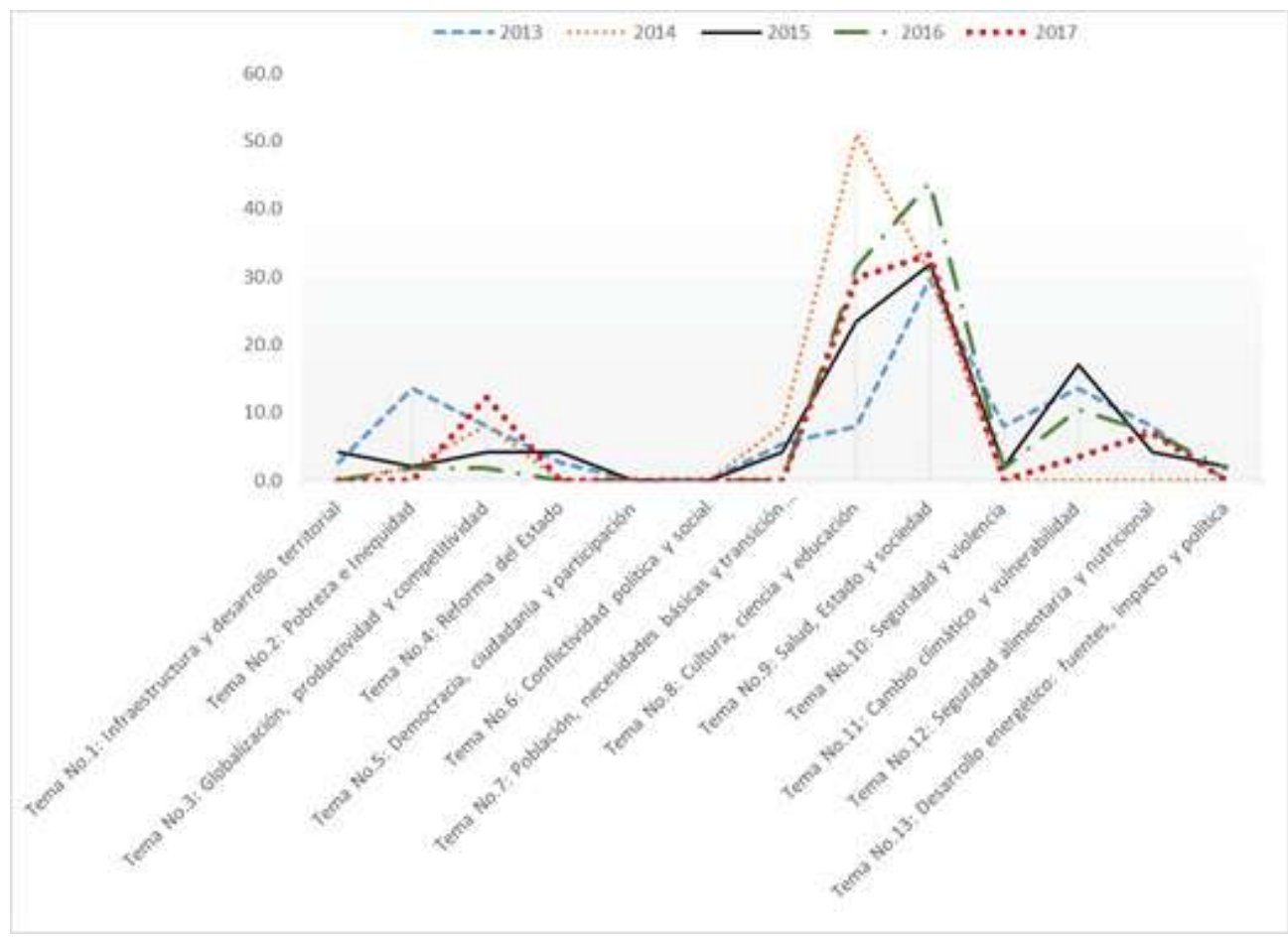

Fuente: Base de datos, DICYP

El tema prioritario No. 9, Salud, Estado y sociedad es el que ha sido mayoritario en tasas de investigaciones durante el período analizado: $29.7 \%$ en $2013,30.6 \%$ en $2014,31.9 \%$ en $2015,43.9 \%$ en 2016 y 38.8 en 2017 . Este tema ha priorizado la 
investigación conducente a la prevención de enfermedades más comunes y la situación del sistema de salud pública. Engloba salud pública, equidad y servicios en salud; endemias, epidemias y respuesta estatal; enfermedades crónicas e infecciosas; enfermedades emergentes y re-emergentes; salud mental y sociedad; poblaciones vulnerables, dependencia y cuidados así como ambiente, nutrición y salud. (DICyP, 2015).

El tema prioritario No. 11, Cambio climático y vulnerabilidad muestra en los años $2013,2015,2016$ y 2017 porcentajes de $13.5 \%, 17.0 \%, 10.5 \%$ y $4.1 \%$ respectivamente. En el año 2014 no se financiaron becas de investigación sobre este tema. Incluye aspectos como impacto del cambio climático en los ecosistemas; monitoreo de la vulnerabilidad; valoración del uso sostenible de los recursos naturales; protección del ambiente (parques y reservas naturales, zonas protegidas y áreas de reserva); manejo de recursos hídricos y saneamiento; impacto ambiental de la transformación del paisaje; evaluación de políticas públicas y esfuerzos colaborativos. (DICyP, 2015)

Los temas sobre los que no se ha solicitado que se financien investigaciones en el período analizado son dos: los No. 5 y 6 . El Tema No. 5 es Democracia, ciudadanía y participación que incluye aspectos como transición, consolidación, calidad y quiebre de la democracia; construcción de ciudadanía y participación política y social; democratización de los espacios locales y regionales; sistema político, electoral y de partidos; legitimidad pública, gestión institucional y satisfacción ciudadana; cultura política y valores democráticos. El otro tema sobre el cual no se ha solicitado fondos es el No. 6 que es Conflictividad política y social que engloba aspectos como conflictividad: causas, manifestaciones y actores; democracia y conflictividad político-institucional y social; gobernabilidad democrática y poderes fácticos; estrategia y mecanismos de prevención y resolución pacífica de conflictos; intervención estatal y solución de conflictos. (DICyP, 2015)

\section{b) Enfoques utilizados en becas de investigación por Facultad y Centros Regio- nales entre 2013 y 2017}

El proceso investigativo tiene por propósito final producir conocimiento por medio de la resolución de un problema de investigación. En este proceso el investigador describe las particularidades de su estudio y la metodología a emplear para el logro de sus objetivos; incluyendo el diseño de la investigación, los métodos a emplear, la población o muestra a estudiar, cómo va a recoger sus datos, etc. 
Las investigaciones han utilizado tres tipos de enfoque:

a) Cuantitativo, que se fundamenta en la construcción y medición de dimensiones, indicadores e índices de variables, y los datos deben responder a estos factores, por lo cual tendrán validez si son verificables o no. (Tamayo y Tamayo, 2014). En este enfoque prevalece la objetividad del investigador, es estructurado, mide las variables y las relaciones que se establecen entre ellas. Se utiliza cuando lo que interesa estudiar es la generalidad de los resultados y la estandarización del conocimiento.

Los investigadores interpretativistas creen que existen múltiples realidades, y que los individuos perciben, entienden, experimentan y dan sentido a la realidad de maneras diferentes según la formación y las experiencias únicas de cada individuo, dando lugar a los paradigmas cualitativos (Pole, 2009).

b) Cualitativo, que se fundamenta en la interpretación, sin proceder a comprobaciones muy rígidas de la realidad objeto de estudio, y permite de forma rápida llegar a situaciones y contextos sociales como grupos y comunidades. Tiene un diseño flexible, donde lo subjetivo e interioridad de los autores y protagonistas se asume como fuente de conocimiento, el cual es entonces un producto social influido por los valores, percepciones y significados de los sujetos que los constituyen (Tamayo y Tamayo, 2014).

c) Con el avance de la ciencia han surgido los equipos multidisciplinarios que introducen en sus investigaciones el uso de las dos metodologías tanto la cuantitativa como la cualitativa convirtiendo las investigaciones en estudios mixtos.

Las investigaciones mixtas son complementarias y proveen diferentes tipos de conocimientos y ventajas al investigador tienen la facilidad para generar y verificar teorías en un mismo estudio, la posibilidad de obtener inferencias más sólidas y la compensación de las desventajas que existen en las metodologías cualitativas y cuantitativas cuando se utilizan individualmente (Ugalde \& Blabastre, 2013).

El enfoque mixto en la investigación utiliza los dos enfoques, siendo uno de ellos preponderante en la investigación y el otro como complemento. Para una utilización óptima de este enfoque los autores deben ser cuidadosos en tenerlo en cuenta no sólo como metodología sino que también en la concepción misma de la teoría, el procesamiento de la información y en los hallazgos y conclusiones. 
Al analizar las becas de investigación realizadas con fondos concursables en la UNAH durante el período 2013-2017 puede observarse en el gráfico 2, que el enfoque más utilizado en las investigaciones con fondos concursables ha sido el cuantitativo, alcanzando $81.6 \%$ en el 2014 , seguido de $75.5 \%$ en el $2017,64.9 \%$ en el 2016 , $56.0 \%$ en el 2013 y $51.1 \%$ en el 2015 .

El enfoque cualitativo es el segundo más utilizado, alcanzando su valor más alto en 2013 (24\%), disminuyendo en el 2014 (10.2\%) y manteniendo el alza en 2015 (19.1\%) y $2016(21.1 \%)$, para bajar en el $2017(8.2 \%)$. El enfoque mixto es el menos utilizado en los años mencionados, teniendo variabilidad en el período: en 2013 fue de 20\%, disminuyendo en 2014 a 8.2\%, aumentando en 2015 (29.8\%) y disminuyendo en 2016 (14.0\%) y subiendo en 2017 (16.3\%).

\section{Gráfico 2. Becas de investigación por tipo de enfoque 2013-2017}

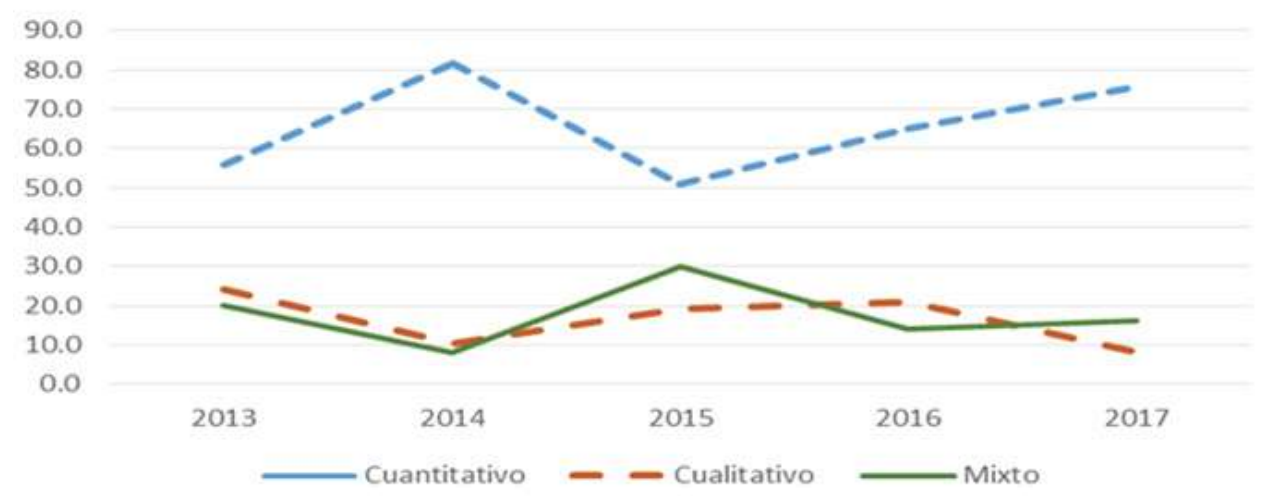

Fuente: Base de datos, DICYP

\section{Enfoque por Unidad Académica}

El siguiente análisis se refiere a los enfoques utilizados por Facultad o Centro Regional en los años 2013 al 2017 que se representan en gráficos individuales para cada año.

La situación en el año 2013 se representa en el gráfico 3. Ahí puede observarse que las facultades de Odontología, Ingeniería y el Centro Universitario Regional de Litoral Atlántico (CURLA), utilizaron en todas las investigaciones sólo el enfoque cuantitativo. Por su parte las Facultades de Ciencias, Ciencias Sociales, Ciencias Económicas, Administrativas y Contables, Ciencias Médicas y Centro Tecnológico de Danlí (UNAH Tec-Danlí) utilizaron además el cualitativo y mixto, siendo mayoritariamente cuantitativo en la Facultad de Ciencias Médicas (80\%). 
El enfoque cualitativo fue utilizado en todas las investigaciones por el Centro Universitario Regional del Centro (CURC), seguido por Ciencias Sociales (71\%), UNAH Tec-Danlí (50\%) y Ciencias Médicas (20\%). El enfoque mixto lo utilizaron las facultades de Ciencias Económicas, Administrativas y Contables (67\%), Ciencias (33\%) y Ciencias Sociales (15\%).

Figura 3. Becas de investigación por Faculta y Centro Regional por tipo de enfoque $2013(\%)$

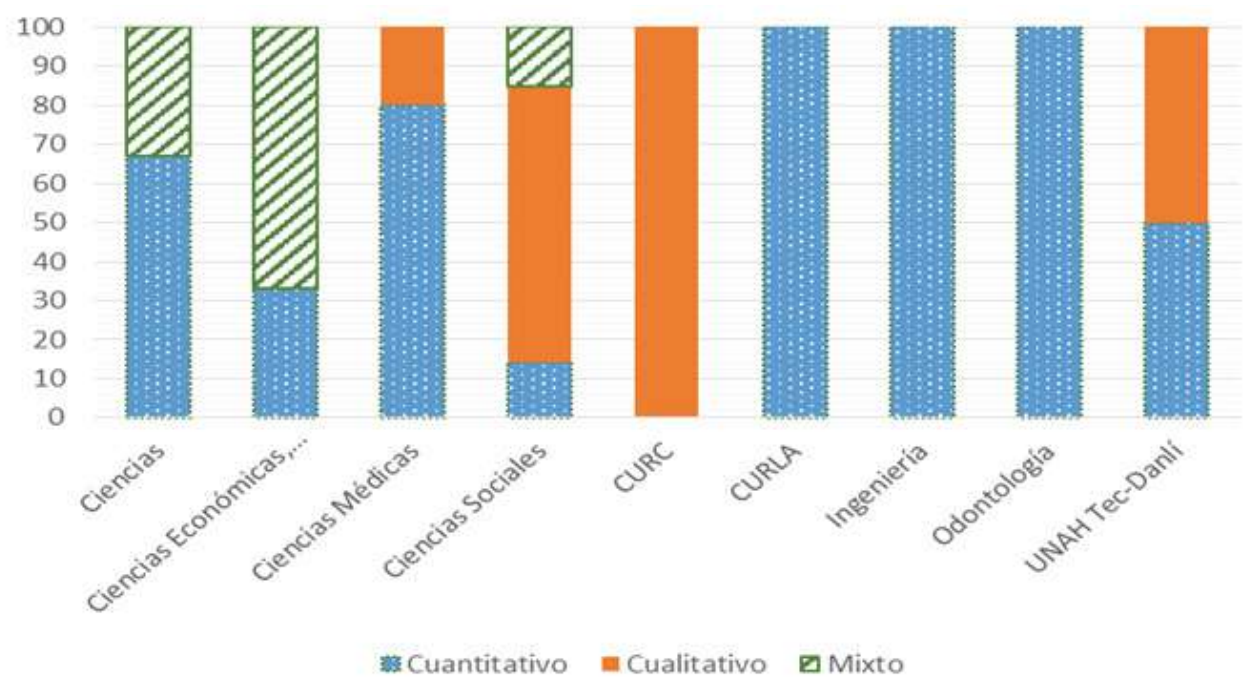

Fuente: Base de datos, DICYP

Puede observarse en el gráfico 4 que en el año 2014 el enfoque cuantitativo se utilizó en todas las investigaciones del Centro Universitario Regional de Occidente (CUROC), Ciencias Espaciales y Ciencias; seguido de Ciencias Económicas, Administrativas y Contables (91.3\%), Ciencias Sociales (83.3\%), Química y Farmacia (75\%), UNAH Tec-Danlí (66.7\%); seguido CURC y Ciencias Médicas (80\%) utilizaron el enfoque cuantitativo en las investigaciones financiadas por la UNAH; siendo Humanidades y Artes la única facultad que no utilizó este enfoque en dicho año. 
Figura 4. Becas de investigación por Faculta y Centro Regional por tipo de enfoque 2014

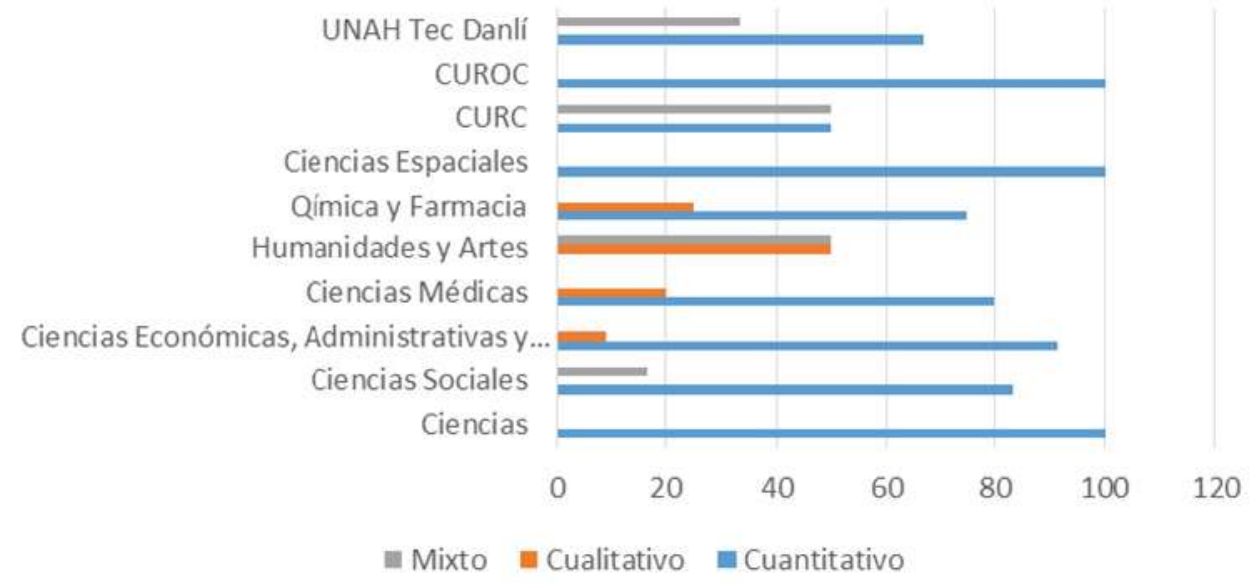

Fuente: Base de datos, DICYP

El enfoque cualitativo fue utilizado en la mitad de las investigaciones de las Facultades de Humanidades y Artes, con el 25\% en Química y Farmacia, Ciencias Médicas $20 \%$, y el $8.7 \%$ en Ciencias Económicas, Administrativas y Contables.

Al analizar el año 2015, se observa en el gráfico No. 5, la Facultad de Ciencias una tendencia a investigar sólo con el enfoque cuantitativo, así como Química y Farmacia, CURLP, Ingeniería y UNAH-VS en un 100\%; Las Facultades Ciencias Espaciales, Ciencias Médicas, y los Centros Regionales CURLA, CUROC y otras unidades lo utilizaron en un $66.7 \%, 50 \%, 50 \%$ y $66.7 \%$ respectivamente.

El enfoque cualitativo en 2015 fue utilizado en 100\% por la facultad de Ciencias Jurídicas. Las Facultades Ciencias Espaciales, Ciencias Sociales, Humanidades y Artes y otras unidades lo utilizaron en $33.3 \%$. 50.0\%, 50.0\% y $75.0 \%$ respectivamente, el Centro Regional CUROC lo utilizó en el tercio de sus investigaciones (33.3\%). En 2015 el enfoque cualitativo lo utilizaron las Facultades Ciencias Médicas, Ciencias Sociales, Humanidades y Artes y el Centro Regional CURLA en $50 \%$ de sus investigaciones financiadas por la UNAH; CURC en el $85.7 \%$.

La Facultad de Ciencias Económicas, Administrativas y Contables y el Tecnológico de Tela utilizaron en todas sus investigaciones en este año el enfoque mixto. 
Figura 5. Becas de investigación por Faculta y Centro Regional por tipo de enfoque 2015

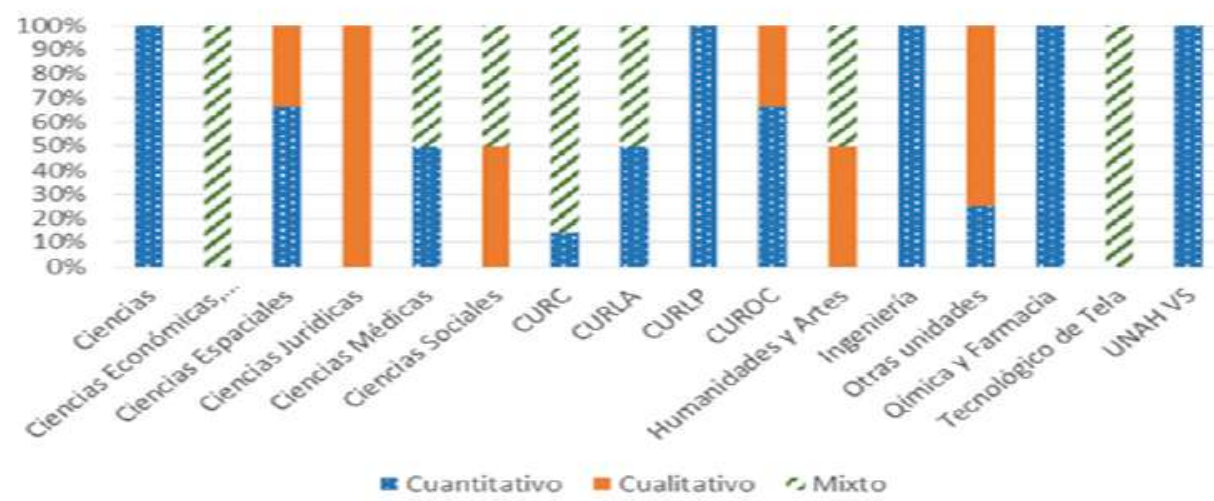

Fuente: Base de datos, DICYP

En 2016 las Facultades Ciencias, Ciencias Económicas, Administrativas y Contables, Ciencias Sociales, Ingeniería, Odontología y Química y Farmacia así como los Centros Regionales CURC, CURLA y CUROC utilizaron este enfoque en el 100\% de sus investigaciones.

Figura 6. Becas de investigación por Faculta y Centro Regional por tipo de enfoque 2016

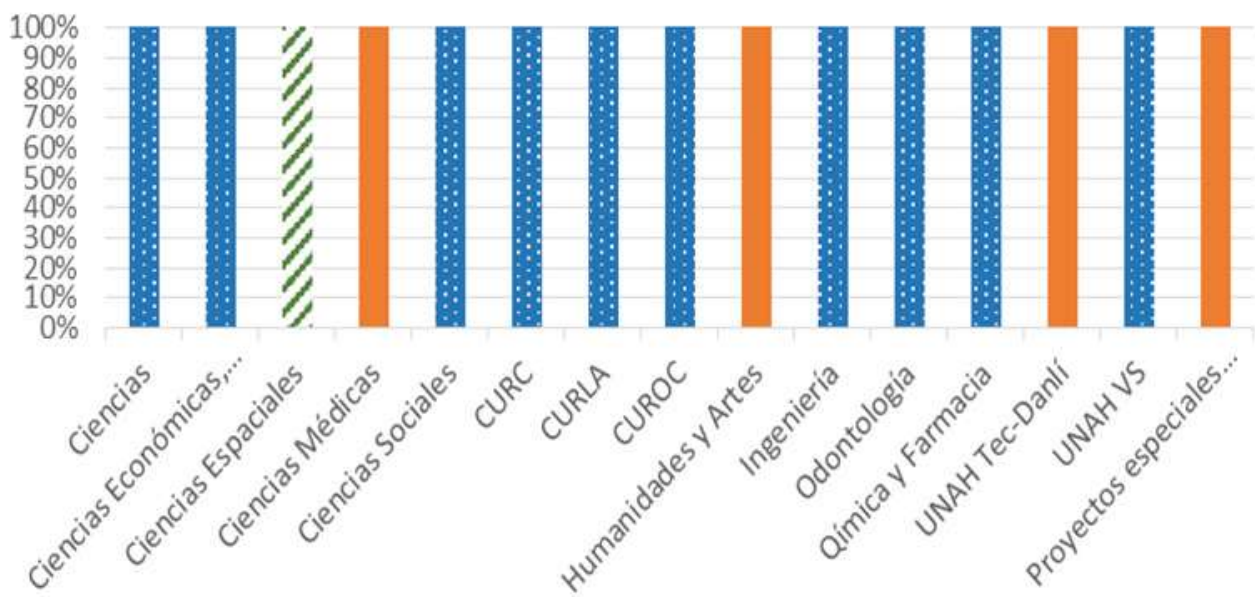

-

Fuente: Base de datos, DICYP 
En el siguiente gráfico se observa que los investigadores que obtuvieron fondos de la UNAH en el año 2017 en los Centros Regionales CURNO, CURC, CURLA, CUROC, CURVA, CURLP enfocaron el 100\% de sus investigaciones con óptica cuantitativa, así como las Facultades de Ciencias Médicas y Odontología.

La Facultad de Ciencias Espaciales muestra la mitad de sus investigaciones en el año 2017 con enfoque cualitativo y la otra mitad mixto; Ciencias Económicas, Administrativas y Contables con el $50 \%$ cuantitativo y $50 \%$ mixto.

Las Facultades y Centros Regionales han optado más frecuentemente con el enfoque cuantitativo en el año 2017, utilizando en segundo lugar el enfoque mixto y en tercer lugar el enfoque cualitativo.

Figura 7. Becas de investigación por Faculta y Centro Regional por tipo de enfoque 2017

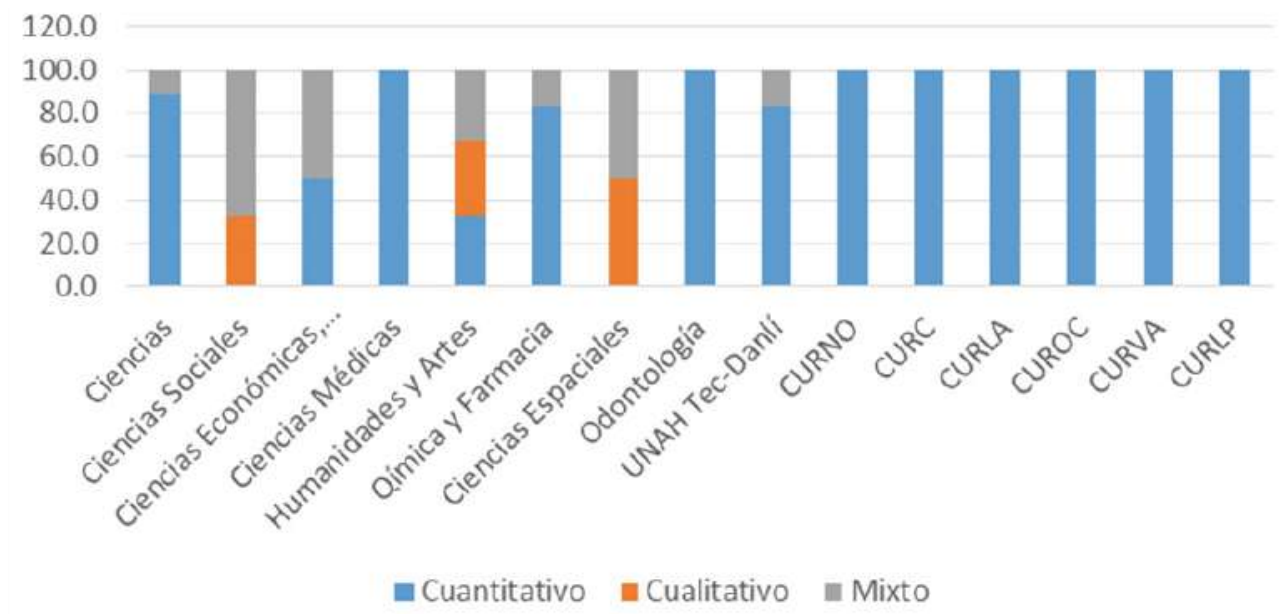

Fuente: Base de datos, DICYP

\section{c) Becas de Investigación por Género}

"Género hace referencia a la construcción social de mujeres y hombres, de feminidad y masculinidad, que varía en el tiempo y el espacio y entre las culturas. La idea de género surgió en los años setenta y fue propuesta por las teorizadoras feministas que desafiaron la posición secundaria de las mujeres en la sociedad". (Ministerio de Ciencia e Innovación de España, 2011) 
En los últimos años las investigaciones han contribuido a conocer realidades y fenómenos inexplorados, a mejorar teorías y conceptos en diferentes campos del conocimiento, a generar nuevos conocimientos para contribuir a resolver problemas de la sociedad. Garantizar la igualdad de oportunidades para hombres y mujeres en la investigación presume una mejor actuación de estos grupos y permite la inclusión de investigadores de alto nivel.

\section{Gráfico 8. Becas de Investigación por género 2013-2017}

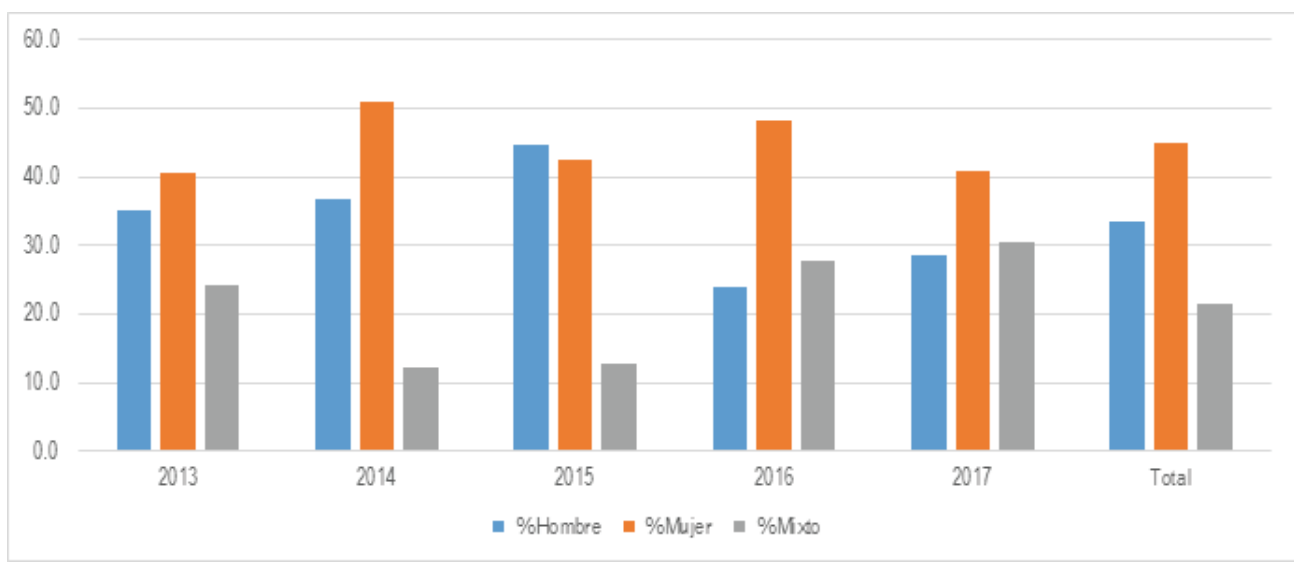

Fuente: Base de datos, DICYP

Si se analizan las investigaciones con fondos concursables en la UNAH durante el periodo comprendido entre 2013 y 2017 por género, se observa que fueron mayoritarias las investigaciones realizadas por mujeres (44.9\%), seguido con el 33.5\% por hombres y el $21.6 \%$ participaron los dos géneros en equipos de investigación. Al observar el gráfico 8, se constata que en el año 2013 el 40.5\% de investigaciones financiadas por la UNAH tuvieron a mujeres como investigadoras, $35.1 \%$ hombres y el $24.3 \%$ mixto. El año 2014 presenta la misma tendencia: el 51\% de mujeres, el $36.7 \%$ de hombres y $12.2 \%$ mixto. En el año 2015 hay bastante similitud entre hombres y mujeres superando los hombres (44.7\%) a las mujeres (42.6\%), siendo el mixto de $12.2 \%$. En el 2016 la mayoría de las investigaciones son realizadas por mujeres $(48.1 \%)$ duplicando el mixto con relación al año anterior $(27.8 \%)$ y los hombres con $24.1 \%$. En el 2017 baja al $40.8 \%$ las mujeres pero siempre manteniéndose a la cabeza, seguido del mixto con $30.6 \%$ y los hombres con $28.6 \%$.

Puede observarse en el gráfico 9, que mayoritariamente las investigaciones fueron realizadas por mujeres en las Facultades de Ciencias Sociales (64\%), Química y Farmacia (63\%), Ciencias (61\%), Ciencias Médicas (50\%), Ciencias Económicas, 
Administrativas y Contables (47\%), y Odontología (43\%); así como en los Centros Regionales CURC (64\%) y UNAH Tec-Danlí (46\%) y el Instituto Tecnológico de Tela con el $100 \%$ de sus investigaciones. ${ }^{2}$

\section{Gráfico 9. Becas de Investigación por género por Facultad y Centro Regional 2013-2017}

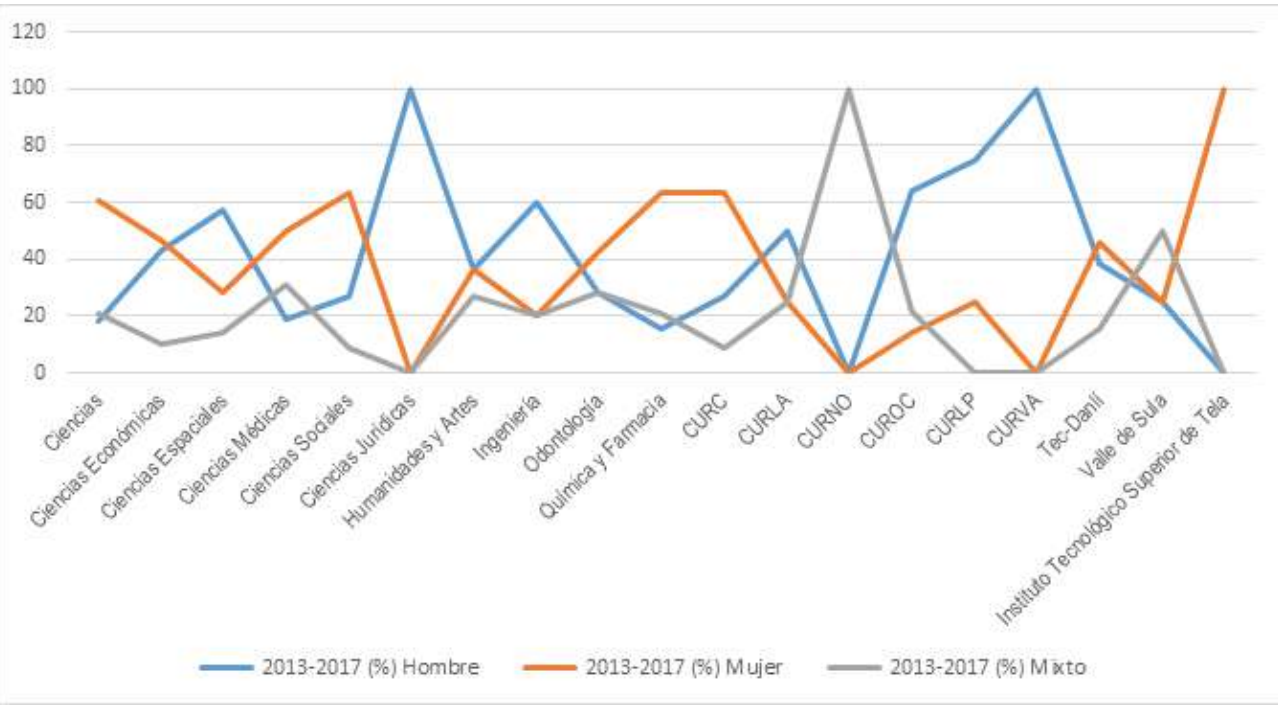

Fuente: Base de datos, DICYP

En el caso de las Facultades o centros Regionales arriba indicados donde las investigaciones realizadas por un género no sobrepasan el $50 \%$, si son mayoritarias al compararlas con los porcentajes de las realizadas por el otro género o por equipos de investigadores de ambos géneros.

Las investigaciones mixtas tienen menor número de investigaciones con relación a las realizadas por hombres y por mujeres individualmente, a excepción en CURNO que tiene el $100 \%$ mixto.

${ }^{2}$ En el caso de las Facultades 0 centros Regionales arriba indicados donde las investigaciones realizadas por un género no sobrepasan el $50 \%$, si son mayoritarias al compararlas con los porcentajes de las realizadas por el otro género o por equipos de investigadores de ambos géneros. 


\section{CONCLUSIONES}

- Las investigaciones financiadas se han enmarcado mayoritariamente en los temas prioritarios 8 y 9 que se refieren a Cultura, ciencia y educación y Salud, Estado y sociedad.

- Los temas prioritarios en los que no se registran investigaciones son los temas prioritarios 5 y 6 que se refieren a Democracia, ciudadanía y participación y conflictividad política y social.

- Las investigaciones financiadas por la UNAH en los años 2013 a 2017 se caracterizan por ser mayoritariamente de enfoque cuantitativo.

- Las Facultades de Ciencias, Ingeniería, Odontología y los Centros Regionales UNAH-VS y CURLP se caracterizan por realizar investigaciones con un enfoque cuantitativo.

- En su mayoría las facultades de Ciencias Económicas, Administrativas y Contables. Química y Farmacia, Ciencias Espaciales y CUROC realizan investigaciones financiadas con enfoque cuantitativo.

- El enfoque cualitativo ha sido utilizado por investigaciones financiadas mayoritariamente por la Facultad de Ciencias Jurídicas.

- Las Facultades de Ciencias Médicas, Ciencias Sociales, Humanidades y Artes y los Centros Regionales CURC, CURLA y UNAH Tec-Danli han utilizado en las investigaciones financiadas los enfoques tanto cuantitativo, cualitativo y mixto.

- El Tecnológico de Tela se caracteriza por realizar investigaciones con un enfoque mixto.

- Las investigaciones financiadas por la UNAH en los años 2013 a 2017 no presentan tendencia en los enfoques utilizados.

- Las investigaciones con fondos concursables en la UNAH en el periodo comprendido entre 2013 y 2017 por género fueron mayoritarias las investigaciones realizadas por mujeres (44.9\%), seguido con el $33.5 \%$ por hombres y el $21.6 \%$ participaron los dos géneros.

- Las investigaciones mixtas tienen menor número de investigaciones en relación a las realizadas por hombres y por mujeres individualmente a excepción de CURNO que tiene el $100 \%$ mixto. 


\section{REFERENCIAS BIBLIOGRÁFICAS}

DICyP, D. d. (2015). Compendios de Investigación Científica. Tegucigalpa, Honduras: UNAH. Ministerio de Ciencia e Innovación de España. (2011). Obtenido de El género en la investigación:http://www.idi.mineco.gob.es/stfls/MICINN/Investigacion/FICHEROS/EI_genero en_la_investigacion.pdf

Pole, K. (2009). Diseño de metodologías mixtas. Una revisión Una revisión de las estrategias para combinar metodologíascuantitativas y cualitativas. Obtenido de Repositorio Institucional del ITESO: https://rei.iteso.mx/bitstream/handle/11117/252/katrhryn_pole.pdf

Tamayo y Tamayo, M. (2014). El proceso de la investigación científica. Mexico: Editorial Limusa, S.A. de C.V.

Ugalde, N., \& Blabastre, F. (2013). Portal de Revistas Académicas, Costa Rica. Obtenido de Investigación cuantitativa e investigación cualitativa:: https://revistas.ucr.ac.cr/index.php/economicas/article/view/12730/11978 